\title{
Osteopontin-c isoform inhibition modulates ovarian cancer cell cisplatin resistance, viability and plasticity
}

\author{
MARIANA CONCENTINO MENEZES BRUM ${ }^{1,2}$, ISABELLA DOS SANTOS GUIMARAES ${ }^{3}$, \\ LUCIANA BUENO FERREIRA ${ }^{1}$, LETICIA BATISTA AZEVEDO RANGEL ${ }^{4}$, \\ RAQUEL CIUVALSCHI MAIA ${ }^{2,5}$, GABRIELA NESTAL DE MORAES ${ }^{2,5^{*}}$ and ETEL R.P. GIMBA ${ }^{1,2,6,7 *}$ \\ ${ }^{1}$ Program of Molecular and Cellular Oncobiology; ${ }^{2}$ Stricto Sensu Graduate Program in Oncology; \\ ${ }^{3}$ Division of Clinical Research, National Institute of Cancer, Rio de Janeiro 20231-050; ${ }^{4}$ Departament of \\ Pharmaceutical Sciences, Federal University of Espírito Santo, Vitória, Espírito Santo 29040-090; \\ ${ }^{5}$ Laboratory of Cellular and Molecular Hemato-Oncology, Program of Molecular Hemato-Oncology, \\ National Institute of Cancer, Rio de Janeiro 20230-130; ${ }^{6}$ Institute of Humanities and Health, \\ Department of Nature Sciences, Fluminense Federal University, Rio das Ostras, Rio de Janeiro 28880-000; \\ ${ }^{7}$ Graduate Program of Biomedical Sciences, Physiology and Pharmacology, \\ Biomedical Institute, Niterói, Rio de Janeiro 24210-130, Brazil
}

Received May 6, 2020; Accepted October 16, 2020

DOI: $10.3892 / o r .2020 .7877$

\begin{abstract}
Osteopontin (OPN) is upregulated in several types of tumor and has been associated with chemoresistance. However, the contribution of OPN splicing isoforms (OPN-SIs) to chemoresistance requires further investigation. The present study aimed to evaluate the expression patterns of each tested OPN-SI in cisplatin (CDDP)-resistant ovarian carcinoma cell lines, focusing on the role of the OPN-c isoform (OPNc) in drug resistance. ACRP ovarian cancer cells resistant to CDDP, as well as their parental cell line A2780, were used. Analyses of the transcriptional expression of OPN-SIs, epithelial-mesenchymal transition (EMT) markers and EMT-related cytokines were performed using reverse transcription-quantitative PCR. OPNc was silenced in ACRP cells using anti-OPNc DNA oligomers and stably overexpressed by transfecting A2780 cells with a mammalian expression vector containing the full length OPNc cDNA. Functional assays were performed to determine cell proliferation, viability and colony formation. The results demonstrated that among the three tested OPN-SIs, OPNc was the most upregulated transcript in the ACRP cells compared with the parental A2780 cells. In addition, the
\end{abstract}

Correspondence to: Dr Etel R.P. Gimba, Institute of Humanities and Health, Department of Nature Sciences, Fluminense Federal University, 1-7 Rua Recife, Bairro Bela Vista, Rio das Ostras, Rio de Janeiro 28880-000, Brazil

E-mail: etelgimba@id.uff.br

${ }^{*}$ Contributed equally

Key words: osteopontin, drug resistance, protein isoforms, osteopontin-c, epithelial-mesenchymal transition, ovarian neoplasms expression levels of P-glycoprotein multidrug transporter were upregulated in CDDP-resistant ACRP cells compared with those in A2780 cells. OPNc knockdown sensitized ACRP cells to CDDP treatment and downregulated P-gp expression levels compared with those in the negative control group. Additionally, silencing of OPNc impaired cell proliferative and colony formation abilities, as well as reversed the expression levels of EMT markers and EMT-related cytokines compared with those in the negative control cells. Notably, although stable OPNc overexpression resulted in increased A2780 cell proliferation, it notably increased CDDP sensitivity compared with that in the cells transfected with a control vector. These results suggested that OPNc silencing may represent a putative approach to sensitize resistant ovarian cancer cells to chemotherapeutic agents.

\section{Introduction}

Ovarian carcinoma, which is one of the most common gynecological cancers, is the seventh most lethal malignancy worldwide (1). The efficacy of platinum-based therapy such as cisplatin (CDDP) in ovarian carcinoma is hindered by the occurrence of drug resistance, a phenomenon often associated with an increased metastatic potential $(2,3)$. Shedding light onto the mechanisms of chemoresistance may render insights into novel therapeutic targets and improved treatment strategies (2-5).

A number of aberrantly expressed gene products mediate tumor chemoresistance, including the upregulation of ATP-binding cassette transporters, such as P-glycoprotein (P-gp) (6). Additional mechanisms, including apoptosis evasion and tumor cell survival, may also contribute to CDDP resistance, among other cancer hallmarks, including tumor cell heterogeneity, redundancy of growth-promoting pathways, increased mutation rate and/or epigenetic alterations (4). 
Similarly, a process termed the epithelial-mesenchymal transition (EMT) has been demonstrated to contribute to drug resistance (7-9). A recent study has also reported that interleukins produced by the tumor microenvironment promote the EMT through altered expression of several inducers of this process, including Snail and STAT transcription factors (5).

Osteopontin (OPN) emerges in the interface between chemoresistance and EMT; it is a matrix glycophosphoprotein that serves important roles in tumor cell proliferation and chemotherapy resistance (10-15). The OPN primary transcript undergoes alternative splicing, generating at least three main splicing isoforms (OPN-SIs), termed OPNa, OPNb and OPNc (16). In addition, OPN is subjected to post-translational modifications, generating several additional isoforms $(17,18)$. The sum of all these isoforms comprises total OPN (tOPN). Although tOPN has been implicated in cancer cell resistance to a wide range of anticancer agents (10), the underlying molecular mechanisms through which the full-length OPN and each OPN-SI specifically perform their roles in chemoresistance remain unclear.

Nakamura et al (19) have demonstrated that prostate cancer cells overexpressing OPNb and OPNc are more resistant to docetaxel compared with cells transfected with an empty vector and exhibit a typical mesenchymal phenotype. Our recent study demonstrated that OPNc was upregulated in distinct B-acute lymphoblastic leukemia (B-ALL) cell lines (20). Our other previous study revealed that OPNc expression levels in B-ALL cells were significantly increased in response to treatment with chemotherapeutic agents that have been used in several backbone treatment strategies for B-ALL, namely vincristine or etoposide (21). Based on these findings, the present study aimed to investigate whether different OPN-SIs may differentially modulate chemoresistance in an ovarian carcinoma cell line model as well as their potential functional roles in the chemoresistant phenotype.

\section{Materials and methods}

Study design. The present study used ACRP, an ovarian cancer cell line resistant to CDDP, as well as its corresponding parental control cell line A2780 as models. Some data obtained using the ACRP cell line have been validated by also testing OVCar-8/Dox ${ }^{\mathrm{R}}$, an ovarian cancer cell line resistant to doxorubicin (Dox), which originated from OVCar-8 cells. Both ovarian cancer cell lines were used to assess the roles of OPNc in chemoresistance. The expression of OPN-SIs and $\mathrm{P}-\mathrm{gp}$ was assessed using reverse transcription-quantitative PCR (RT-qPCR). After evaluating OPNc expression in the CDDP and Dox resistance models, the OPNc isoform was silenced in order to evaluate its roles in the resistant phenotype by transfecting ACRP and OVCar-8/Dox ${ }^{\mathrm{R}}$ cells with a specific anti-OPNc DNA oligomer modified with phosphorothiotates. In these cell lines, functional assays were performed using 3-(4,5-dimethylthiazol-2-yl)-2,5-diphenyltetrazolium bromide (MTT), trypan blue and clonogenic assays. The biological effects were validated by analyzing the mRNA expression levels of EMT markers and cytokines. To validate the cytotoxicity results observed using the knockdown approach, experimental assays were performed in the A2780 parental cell line ectopically overexpressing OPNc $\left(\mathrm{OPNc}^{+}\right)$. OPNc and $\mathrm{P}$-gp expression levels were determined in the $\mathrm{A} 2780 \mathrm{OPNc}^{+}$ cell line, and additional functional assays were performed, including MTT, trypan blue exclusion and clonogenic assays in the absence or presence of CDDP.

Cell lines and culture conditions. The epithelial ovarian cancer cell line A2780 and the corresponding CDDP-resistant cell line ACRP were generously provided by Dr Pat J. Morin (National Institutes of Health, Bethesda, MD, USA). ACRP cells were selected for progressive resistance to CDDP as previously described (22). The cells were maintained in RPMI-1640 medium (Gibco; Thermo Fisher Scientific, Inc.) supplemented with $10 \%$ fetal bovine serum (FBS; Gibco; Thermo Fisher Scientific, Inc.) at $37^{\circ} \mathrm{C}$ in a humidified atmosphere of $5 \% \mathrm{CO}_{2}$. The human ovarian cell line OVCar- 8 was acquired from the American Type Culture Collection. The OVCar-8 cell line resistant to Dox, termed OVCar-8/Dox ${ }^{\mathrm{R}}$ resistant cell line, was originated by progressively culturing OVCar- 8 cells with increasing concentrations of Dox for 6 months. The doses were incrementally increased upon selection of Dox-resistant clones up to $17 \mu \mathrm{M}$ Dox, which was used to maintain the OVCar-8/Dox ${ }^{\mathrm{R}}$ cells.

Isolation of total RNA and RT-qPCR. Total cellular RNA was isolated from the cells using TRIzol ${ }^{\circledR}$ reagent (Invitrogen; Thermo Fisher Scientific, Inc.) according to the manufacturer's protocol. The RNA was reverse-transcribed using SuperScript $^{\mathrm{TM}}$ II Reverse Transcriptase kit (Invitrogen; Thermo Fisher Scientific, Inc.) according to the manufacturer's protocol. mRNA expression analysis was performed by qPCR using the Eco Real-Time PCR System (Illumina, Inc.) and Power SYBR ${ }^{\circledR}$ Green PCR Master mix (Applied Biosystems; Thermo Fisher Scientific, Inc.). The thermocycling conditions were as follows: Initial incubation at $50^{\circ} \mathrm{C}$ for $2 \mathrm{~min}$ and $95^{\circ} \mathrm{C}$ for $10 \mathrm{~min}$, followed by additional incubation at $94^{\circ} \mathrm{C}$ for $5 \mathrm{~min} ; 40$ cycles of $94^{\circ} \mathrm{C}$ for $30 \mathrm{sec}, 60^{\circ} \mathrm{C}$ for $30 \mathrm{sec}$ and $72^{\circ} \mathrm{C}$ for $45 \mathrm{sec}$; and final melting curve analysis at $72^{\circ} \mathrm{C}$ for $15 \mathrm{sec}$, $90^{\circ} \mathrm{C}$ for $5 \mathrm{sec}, 55^{\circ} \mathrm{C}$ for $5 \mathrm{sec}$ and $90^{\circ} \mathrm{C}$ for $5 \mathrm{sec}$. Fold-changes in the expression levels of each mRNA was calculated using the $2^{-\Delta \Delta C q}$ method (23). GAPDH or $\beta$-actin were used as the normalization controls. The PCR primers sequences are listed in Table I.

OPNc knockdown using anti-OPNc oligomers. After reaching 90-95\% confluency, ACRP or OVCar- 8 Dox $^{\mathrm{R}}$ cells were transfected with $100 \mathrm{nM}$ anti-OPNc phosphorothiotate-modified antisense DNA oligomer (ASO anti-OPNc; 5'-A* $\mathrm{C}^{*} \mathrm{~A}^{*} \mathrm{AC} \mathrm{C}^{*} \mathrm{GC}$ ATTCTGCTTT $\left.* \mathrm{~T}^{*} \mathrm{C} * \mathrm{C}-3^{\prime}\right)$ or with a non-specific/scrambled control sequence (ASO SCR; 5'-C*C*T*T*TTCGTCTTACGA $C^{*} A^{*} C^{*} A-3 '$ ), using Lipofectamine ${ }^{\circledR} 2000$ (Invitrogen; Thermo Fisher Scientific, Inc.) according to the manufacturer's instructions. The phosphorothiotate-modified bases are marked by an asterisk in the ASO sequences. Transfections were performed with $24 \mu \mathrm{g}$ oligomers and $24 \mu \mathrm{l}$ Lipofectamine ${ }^{\circledR} 2000$ diluted in RPMI-1640 medium and Opti-MEM in $10-\mathrm{cm}$ plates in a $5 \% \mathrm{CO}_{2}$ incubator at $37^{\circ} \mathrm{C}$ for $24 \mathrm{~h}$. The transfected cells were collected at $24 \mathrm{~h}$ post-transfection for subsequent experiments. The downregulation of the OPNc mRNA expression levels was confirmed by RT-qPCR. 
Table I. Forward and reverse oligonucleotide sequences.

\begin{tabular}{|c|c|}
\hline Gene & Sequence $\left(5^{\prime} \rightarrow 3^{\prime}\right)$ \\
\hline \multirow[t]{2}{*}{ P-gp } & F: CCCATCATTGCAATAGCAGG \\
\hline & R: GTTCAAACTTCTGCTCCTGA \\
\hline \multirow[t]{2}{*}{$\mathrm{OPNa}$} & F: ATCTCCTAGCCCCACAGAAT \\
\hline & R: CATCAGACTGGTGAGAATCATC \\
\hline \multirow[t]{2}{*}{$\mathrm{OPNb}$} & F: CTCCTAGCCCCACAGACCCT \\
\hline & R: TATCACCTCGGCCATCATATG \\
\hline \multirow[t]{2}{*}{ OPNc } & F: CTGAGGAAAAGCAGAATG \\
\hline & R: AATGGAGTCCTGGCTGT \\
\hline \multirow[t]{2}{*}{ GAPDH } & F: TCCCATCACCATCTTTCAGGAGCA \\
\hline & R: TTCTACATGGTGGTGAAGACGCCA \\
\hline \multirow[t]{2}{*}{$\beta$-actin } & F: GGCGGCACCACCATGTACCCT \\
\hline & R: AGGGGCCGGACTCGTCATACT \\
\hline \multirow[t]{2}{*}{ E-cadherin } & F: GAATGACAACAAGCCCGAAT \\
\hline & R: GAC CTCCATCACAGAGGTTCC \\
\hline \multirow[t]{2}{*}{ Cytokeratin-18 } & F: GCGAGAAGGAGACCATGCA \\
\hline & R: GGTGTTCCCGGATTTTGATCT \\
\hline \multirow[t]{2}{*}{ Vimentin } & F: GACAATGCGTCTCTGGCACGTCTT \\
\hline & R: TCC TCCGCCTCCTGCAGGTTCTT \\
\hline \multirow[t]{2}{*}{ N-cadherin } & F: GGTGGAGGAGAAGAAGACCAG \\
\hline & R: GCA TCAGGCTCCACAGT \\
\hline \multirow[t]{2}{*}{ Claudin-3 } & F: CTGCTCTGCTGCTCGTGTCC \\
\hline & R:TTAGACGTAGTCCTTGCGGTCGTAG \\
\hline \multirow[t]{2}{*}{ Slug } & F: TTCGGACCCACACATTACCT \\
\hline & R: GCAGTGAGGGCAAGAAAAAG \\
\hline \multirow[t]{2}{*}{ Snail } & F: TTCCAGCAGCCCTACGACCAG \\
\hline & R: CTTTCCCACTGTCCTCATC \\
\hline \multirow[t]{2}{*}{ Twist } & F: CCCAACTCCCAGACACCTC \\
\hline & R: CAAAAAGAAAGCGCCCACC \\
\hline \multirow[t]{2}{*}{ IL-6 } & F: CATTTGTGGTTGGGTCAGG \\
\hline & R: AGTGAGGAACAAGCCAGAGC \\
\hline \multirow[t]{2}{*}{ IL-8 } & F: CTTGGCAGCCTTCCTGATTT \\
\hline & R: GGGTGGAAAGGTTTGGAGTATG \\
\hline \multirow[t]{2}{*}{ IL1- $\alpha$} & F: CATCCTCCACAATAGCAGACAG \\
\hline & R: GAGTTTCCTGGCTATGGGATAAG \\
\hline \multirow[t]{2}{*}{ IL1- $\beta$} & F: CAAAGGCGGCCAGGATATAA \\
\hline & R: CTAGGGATTGAGTCCACATTCAG \\
\hline \multirow[t]{2}{*}{ GP130 } & F: TGCCTCCAGAAAAACCTAAAAA \\
\hline & R: TTTGTCTCCAAGTGTGTTTCC \\
\hline
\end{tabular}

OPN, osteopontin; P-gp, P-glycoprotein; F, forward; R, reverse.

OPNc overexpression. To generate a cell line stably overexpressing OPNc (A2780 $\mathrm{OPNc}^{+}$), 4x105 A2780 cells were plated in 6-well plates and maintained for $24 \mathrm{~h}$ in a $5 \% \mathrm{CO}_{2}$ incubator at $37^{\circ} \mathrm{C}$. Following adhesion, the cells were transfected with $2 \mu \mathrm{g}$ pCR3.1 expression plasmid containing the complete cDNA encoding OPNc or with the empty vector plus $4 \mu$ l Lipofectamine ${ }^{\circledR}$ 2000, which were diluted in RPMI-1640 medium and Opti-MEM. The cells were then maintained in a $5 \% \mathrm{CO}_{2}$ incubator at $37^{\circ} \mathrm{C}$ for $24 \mathrm{~h}$. Subsequently,
$300 \mu \mathrm{g} / \mathrm{ml}$ geneticin was added to the culture medium to select the transfected clones. The culture medium containing $300 \mu \mathrm{g} / \mathrm{ml}$ geneticin was changed every 2 days until clones stably expressing the OPNc isoform were selected, which lasted 15 days. Subsequent experiments were performed following the selection or $\mathrm{A} 2780 \mathrm{OPNc}^{+}$cell line in selection culture media. Following selection, A2780 $\mathrm{OPNc}^{+}$and A2780 pCR3.1 cells were routinely maintained with $300 \mu \mathrm{g} / \mathrm{ml}$ geneticin to ensure OPNc overexpression.

MTT assay. The MTT assay (Uniscience Corp.) was used to measure the cytotoxic effects of CDDP (Libbs Farmaceutica, Ltda.) on ovarian cancer cell lines. Briefly, ACRP, OVCar-8 $\mathrm{Dox}^{\mathrm{R}}, \mathrm{A} 2780 \mathrm{OPNc}^{+}$cells or A2780 cells transfected with the pCR3.1 empty vector were seeded in 96-well plates $\left(1 \times 10^{4}\right.$ cells/well). After adhesion, the cells were exposed to 10 , 20, 40, 60 and $100 \mu \mathrm{M}$ CDDP or drug-free complete medium for 24 or $96 \mathrm{~h}$. Drug-free medium was added to the control wells. A total of $20 \mu \mathrm{l} \mathrm{MTT}(5 \mathrm{mg} / \mathrm{ml})$ was added to each well $4 \mathrm{~h}$ prior to the end of the time intervals. MTT solution was then removed at 24 or $96 \mathrm{~h}$, and the formazan crystals were dissolved in $150 \mu \mathrm{l}$ DMSO (Sigma-Aldrich; Merck KGaA). The optical density at $570 \mathrm{~nm}$ was determined using a SpectraMax 190 microplate reader. All experiments were performed in triplicate.

Clonogenic assay. To determine clonogenicity, a total of $2 \times 10^{3}$ ACRP, OVCar- 8 Dox $^{\mathrm{R}}, \mathrm{A} 2780 \mathrm{OPNc}^{+}$cells or A2780 cells transfected with the pCR3.1 empty vector were seeded in 6-well plates and maintained in culture for $\sim 14$ days, until colonies could be observed. Colonies were fixed in absolute ethanol for $10 \mathrm{~min}$ and stained with $0.5 \%$ crystal violet (Sigma-Aldrich; Merck KGaA) for $1 \mathrm{~h}$ at room temperature. The crystals were dissolved in $33 \%$ glacial acetic acid solution, and the optical density was measured at $595 \mathrm{~nm}$ using a SpectraMax 190 microplate reader.

Statistical analysis. Data are presented as the mean \pm SD of three independent experiments. Differences in various parameters between two groups were analyzed by two-tailed Student's t-test. GraphPad Prism version 5.02 (GraphPad Software, Inc.) was used to plot the graphs. Statistical analysis was performed using Microsoft Excel 2010 (Microsoft Corporation). $\mathrm{P}<0.05$ was considered to indicate a statistically significant difference.

\section{Results}

OPNc is upregulated in ACRP chemoresistant cells. As a first approach to determine whether the three tested OPN-SIs differently modulated CDDP resistance in ovarian cancer cell lines, the transcriptional levels of each OPN-SI were analyzed in ACRP cells and compared with those in the corresponding parental cells. Among the three tested OPN-SIs, only OPNc expression levels were upregulated in ACRP cells compared with those in the parental A2780 cell line, although the results were not statistically significant (Fig. 1A).

Since tOPN can modulate P-gp expression (6), P-gp expression was subsequently tested in ACRP cells. The results demonstrated that the mRNA expression levels of P-gp were also upregulated in ACRP cells compared with those in the 

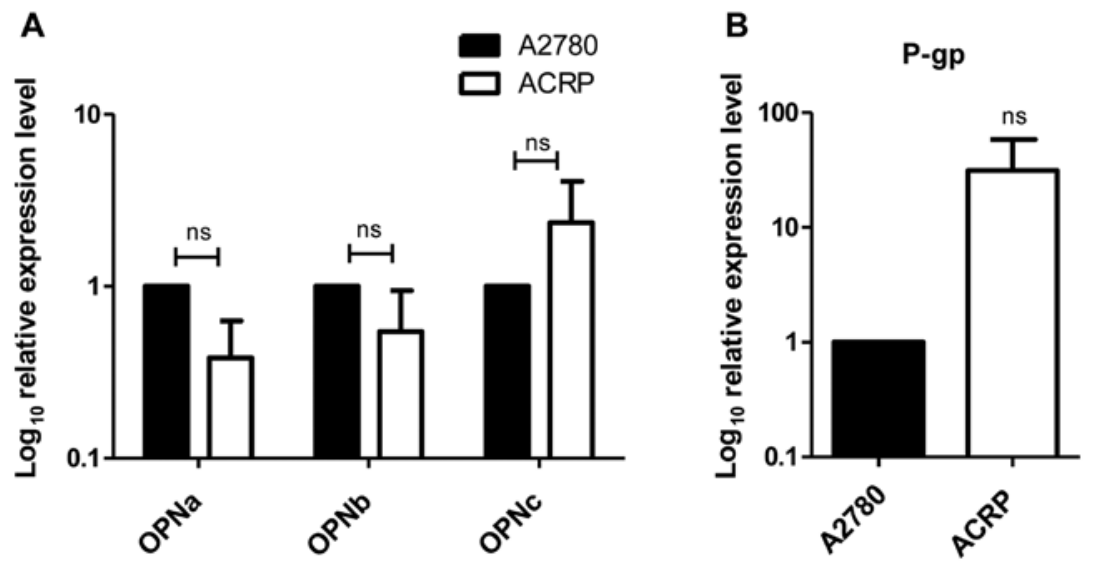

Figure 1. OPNc and P-gp are non-significantly upregulated in cisplatin-resistant ACRP ovarian cancer cells. (A) RT-qPCR was used to analyze the mRNA expression levels of OPNa, OPNb and OPNc isoforms. (B) P-gp mRNA expression levels were analyzed in ACRP cells by RT-qPCR. Log $_{10}$ relative expression levels were calculated using the $2^{-\Delta \Delta C q}$ method and normalized to those of $\beta$-actin. ns, non-significant. OPN, osteopontin; RT-qPCR, reverse transcription-quantitative PCR; P-gp, P-glycoprotein.

parental control cells, although the results did not reach statistical significance (Fig. 1B).

OPNc knockdown sensitizes ACRP cells to CDDP treatment. To further characterize the involvement of OPNc in CDDP-resistant ACRP cells, the OPNc isoform was knocked down using the ASO anti-OPNc. OPNc mRNA expression levels were reduced compared with those in cells transfected with the ASO-SCR (Fig. 2A). By contrast, OPNc knockdown in ACRP cells resulted in the upregulation of OPNa and OPNb mRNA expression levels compared with those in the ASO-SCR group, although this result did not achieve statistical significance (Fig. S1). Notably, following OPNc silencing, P-gp expression levels were also downregulated compared with those in cells transfected with the ASO-SCR (Fig. 2B), suggesting that OPNc may modulate P-gp expression.

The present study then tested whether OPNc knockdown may modulate ACRP cell viability in response to CDDP exposure. Following OPNc knockdown, ACRP cells were more sensitive to 10 and $40 \mathrm{mM}$ CDDP treatment compared with the ASO SCR-transfected cells $24 \mathrm{~h}$ after drug treatment (Fig. 2C and D).

To further determine whether the roles of OPNc in drug resistance were dependent on the cell line model and the class of chemotherapeutic drug, the same experiments were performed in the OVCar- 8 Dox $^{\mathrm{R}}$ cell line. The results demonstrated a strong but non-significant downregulation of the P-gp expression levels following OPNc knockdown in these cells compared with those in cells transfected with the ASO SCR (Fig. S2A and B), which was similarly associated with Dox sensitization following $24-\mathrm{h}(0.05$ and $0.1 \mu \mathrm{M})$ and $96-\mathrm{h}(0.1$, $0.2,0.5$ and $1 \mu \mathrm{M}$ ) drug exposure (Fig. S2C and D). These results suggested that OPNc may modulate drug resistance and cell viability in distinct models of drug-resistant ovarian cancer cells.

OPNc modulates cell viability and colony formation in ACRP cells. The effects of OPNc knockdown on the chemoresistant ovarian cancer cell line viability were next assessed. Notably, the number of ACRP cells was decreased in response to OPNc silencing compared with that of the ASO SCR-transfected cells (Fig. 2E). The colony formation capacity of ACRP cells transfected with the ASO anti-OPNc was analyzed, and the results demonstrated that OPNc knockdown reduced the colony formation capacity in ACRP cells compared with that of the control group, but the difference was not statistically significant (Fig. 2F). Similarly, the cell number and clonogenicity were impaired in OVCar- 8 Dox ${ }^{\mathrm{R}}$ cells transfected with the ASO anti-OPNc compared with those in the control cells (Fig. S3A and B). These results suggested that OPNc may not only modulates drug sensitivity, but also promote cell viability.

OPNc affects the expression of EMT markers in ACRP cells. The present study further investigated whether OPNc may modulate the EMT, and the RT-qPCR results demonstrated that ACRP cells exhibited an intermediate or partial EMT phenotype compared with that of the parental A2780 cell line. In ACRP cells, the expression levels of the epithelial markers E-cadherin, claudin-3 and cytokeratin-18 were upregulated compared with those in A2780 cells (Fig. 3A). In addition, vimentin and $\mathrm{N}$-cadherin levels were also upregulated, whereas Slug, Snail and Twist exhibited similar or lower expression levels compared with those in A2780 cells (Fig. 3B). However, none of these differences were statistically significant. In response to OPNc silencing, changes in the expression patterns of the epithelial and mesenchymal markers were observed. E-cadherin expression levels were non-significantly upregulated in ACRP cells following OPNc silencing compared with those in the control cells. By contrast, the levels of claudin-3 and cytokeratin-18 were non-significantly downregulated in the same experimental conditions (Fig. 3C). Among the mesenchymal markers, the vimentin and $\mathrm{N}$-cadherin transcriptional levels remained largely unchanged following OPNc silencing in ACRP cells, whereas the levels of Slug, Snail and Twist transcripts were downregulated compared with those in the ASO SCR-transfected cells (Fig. 3D). These results demonstrated alterations in the EMT marker transcriptional patterns in response to OPNc downregulation in ACRP resistant cell line, suggesting that OPNc may modulate CDDP resistance-associated EMT in these cells. 


\section{A}
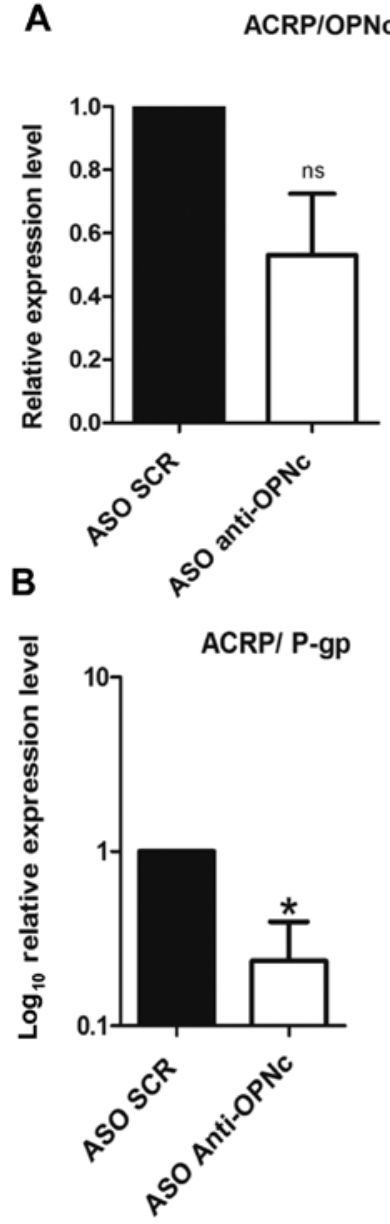

E

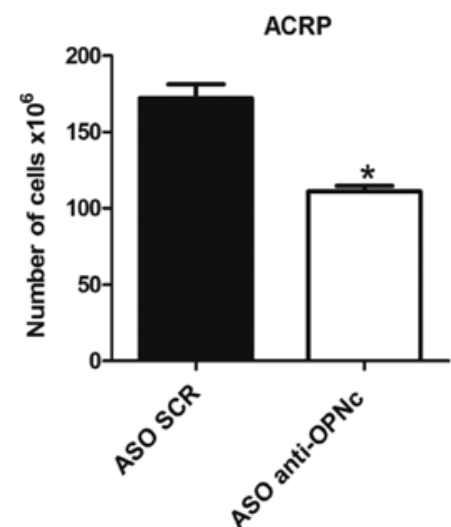

C
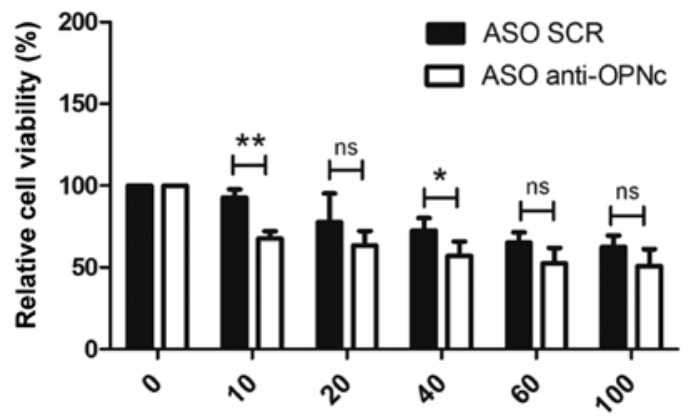

D

Cisplatin ( $\mu \mathrm{M}), 24 \mathrm{~h}$

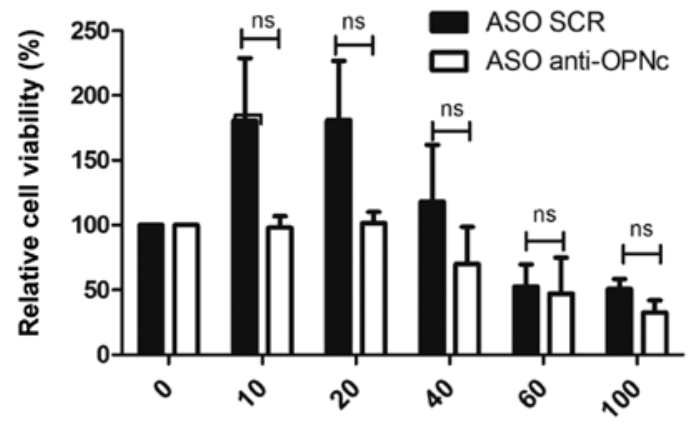

Cisplatin $(\mu \mathrm{M}), 96 \mathrm{~h}$

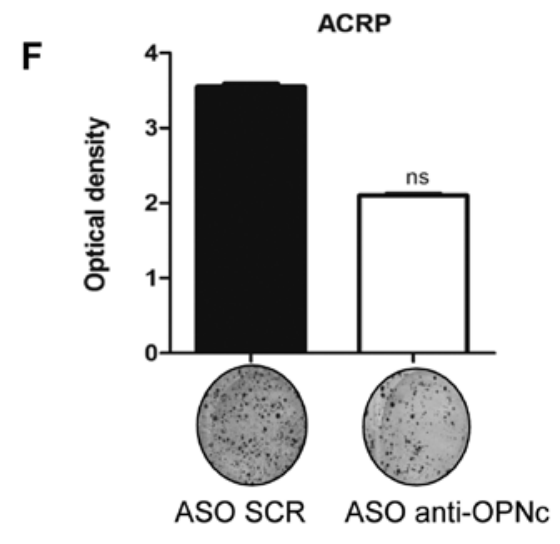

Figure 2. Knockdown of OPNc downregulates P-gp mRNA expression levels and sensitizes ACRP cells to cisplatin, impairing cell viability and colony formation. (A and B) ACRP cisplatin-resistant ovarian cancer cells were transfected with ASO SCR or ASO anti-OPNc, and reverse transcription-quantitative PCR was used to determine (A) OPNc and (B) P-gp mRNA expression levels. Relative expression levels were calculated using the $2^{-\Delta \Delta C q}$ method and normalized to those of $\beta$-actin. (C and D) At $24 \mathrm{~h}$ post-transfection, ACRP cells were treated with increasing concentrations of cisplatin for (C) 24 and (D) $96 \mathrm{~h}$, and cell viability was measured by the MTT assay. (E) Cell viability was also evaluated by trypan blue exclusion analysis at $24 \mathrm{~h}$ post-transfection. (F) ACRP cells were assessed for clonogenicity following 14 -day culture by crystal violet staining. Optical density was measured at $595 \mathrm{~nm}$. ${ }^{*} \mathrm{P} \leq 0.05$ and ${ }^{* *} \mathrm{P} \leq 0.01$; $\mathrm{ns}$, non-significant. OPNc, osteopontin-c isoform; ASO, antisense oligonucleotide; SCR, scramble; P-gp, P-glycoprotein.

OPNc affects the expression of EMT-related cytokines. The present study next tested the expression levels of the EMT-related cytokines IL-6, IL-8, IL-1 $\alpha$, IL- $1 \beta$ and the GP130 receptor. Notably, a general non-significantly upregulated expression pattern was observed for the EMT-related cytokines in ACRP cells compared with that in the parental cell line A2870 (Fig. 3E). Consistently, the expression levels of IL-6, IL-8 and IL-1 $\alpha$ were significantly downregulated, and the levels of IL-1 $\beta$ and the GP130 receptor were non-significantly downregulated in the OPNc-silenced ACRP cells compared with those in the cells transfected with the ASO SCR (Fig. 3F). In addition, decreased levels of EMT-associated cytokines were observed in OVCar-8 Dox ${ }^{\mathrm{R}}$ cells following OPNc knockdown compared with those in ASO SCR-transfected control cells (Fig. S4), further suggesting that OPNc may be associated with drug resistance as well as with the EMT phenotype. 

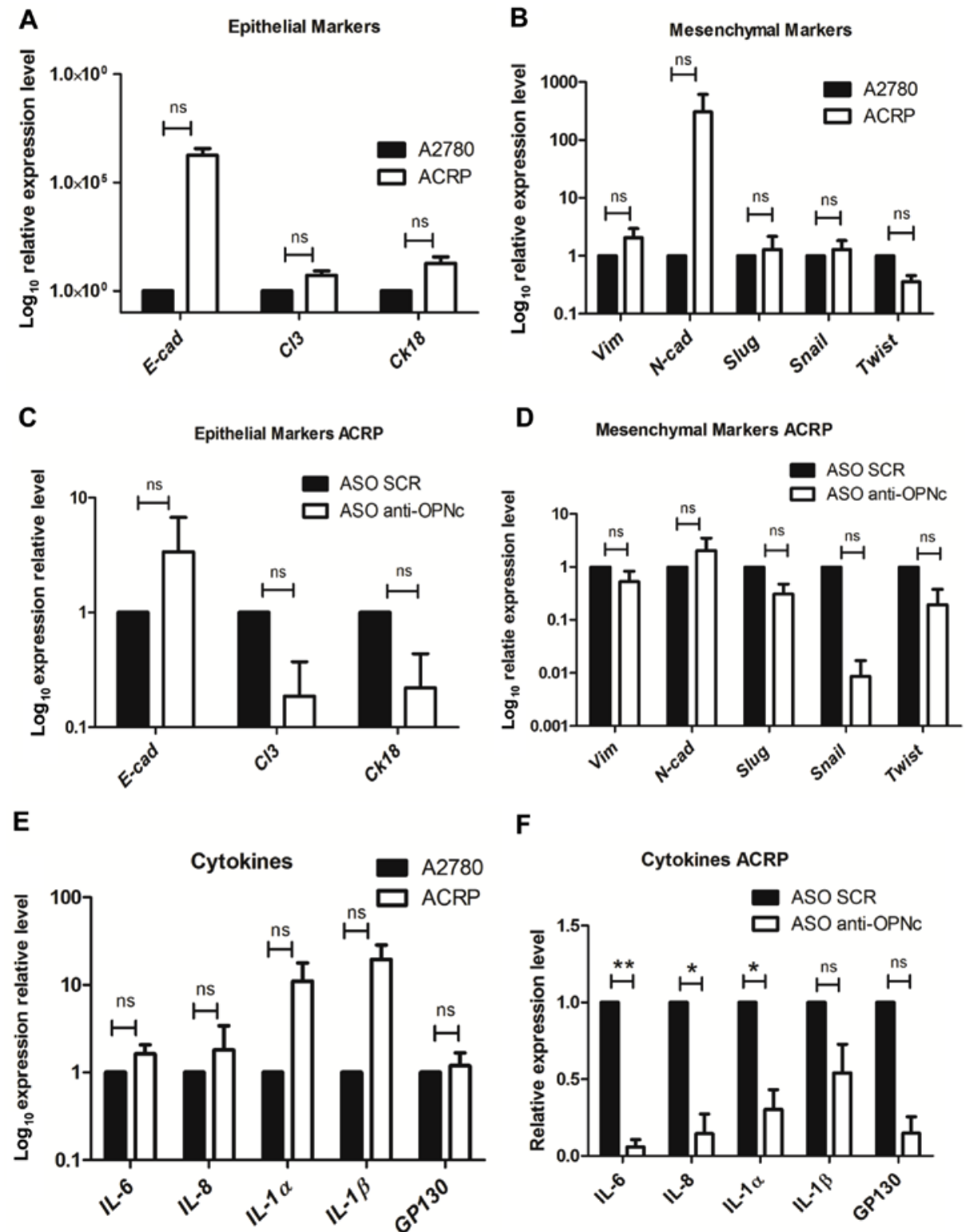

$\mathbf{F}$

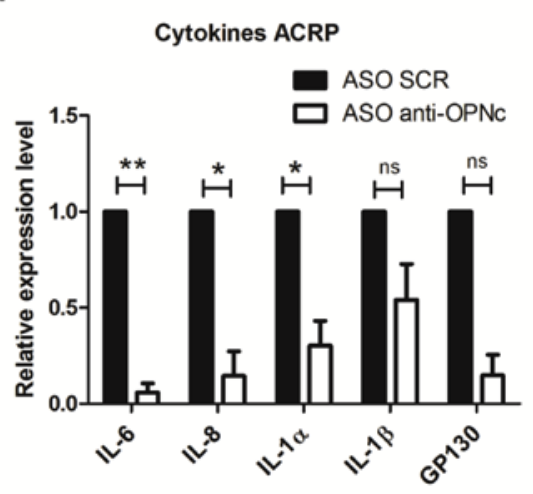

Figure 3. OPNc expression levels modulate the epithelial-mesenchymal transition marker and associated interleukin expression patterns in cisplatin-resistant ACRP ovarian cancer cells. (A, B and E) A2780 and ACRP cells were harvested, and the expression levels of (A) epithelial and (B) mesenchymal markers, as well as (E) IL-6, IL-8, IL-1 $\alpha$, IL-1 $\beta$ and the GP130 receptor were analyzed by RT-qPCR. (C, D and F) ACRP cells were transfected with ASO SCR or ASO anti-OPNc, and RT-qPCR was used to determine the mRNA expression levels of (C) epithelial and (D) mesenchymal markers, as well as (F) IL6, IL8, IL-1 $\alpha$, IL-1 $\beta$ and the GP130 receptor. The mRNA expression levels of E-cad, Cl3, ck18, Vim, N-cad, Slug, Snail and Twist were normalized to those of GAPDH, and the expression levels of IL6, IL8, IL-1 $\alpha$, IL-1 $\beta$ and the GP130 receptor were normalized to those of $\beta$-actin. Relative expression levels were calculated using the $2^{-\Delta \Delta C q}$ method. ${ }^{*} \mathrm{P} \leq 0.05$ and ${ }^{* *} \mathrm{P} \leq 0.01$; ns, non-significant. OPNc, osteopontin-c isoform; RT-qPCR, reverse transcription-quantitative PCR; ASO, antisense oligonucleotide; SCR, scramble; E-cad, E-cadherin; Cl3, claudin-3; ck18, cytokeratin-18; Vim, vimentin; N-cad, N-cadherin.

$O P N c^{+}$cells exhibit enhanced proliferative capacity and sensitivity to CDDP. To further determine the cellular effects of OPNc, this splice variant was stably overexpressed in the A2780 parental cells (Fig. 4A). The results demonstrated that the $\mathrm{OPNc}^{+}$cells also displayed high P-gp expression levels (Fig. 4B). Notably, OPNc overexpression improved the cell proliferative capacity, as denoted by a higher number of cells (Fig. 4C), cell viability at $96 \mathrm{~h}$ (Fig. 4D) and clonogenic potential (Fig. 4E) in the $\mathrm{OPNc}^{+}$group compared with those in the empty vector-transfected control group.

In order to address whether OPNc overexpression modulated the response to $\mathrm{CDDP}, \mathrm{OPNc}^{+}$cells were exposed to increasing CDDP concentrations for 24 and 96 h. Notably,
OPNc+ cells became more susceptible to CDDP treatment compared with cells transfected with the empty PCR3.1 expression vector after 24-h drug treatment (Fig. 5A). These results were confirmed in a long-term assessment of cell viability, which demonstrated that $\mathrm{OPNc}+$ cells formed fewer colonies in response to CDDP treatment (Fig. 5B). We then hypothesized that CDDP may negatively modulate the expression of OPNc, further sensitizing $\mathrm{OPNc}^{+}$cells to this drug. The results of RT-qPCR analysis demonstrated that CDDP downregulated the OPNc mRNA levels in $\mathrm{OPNc}^{+}$cells (Fig. 5C) following 24-h CDDP treatment. However, these effects were less pronounced following 96-h CDDP exposure (Fig. S5A and B). These results demonstrated that CDDP 
A

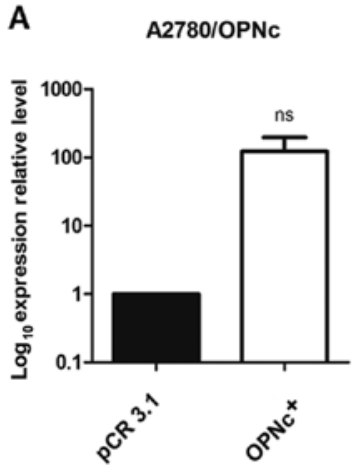

C

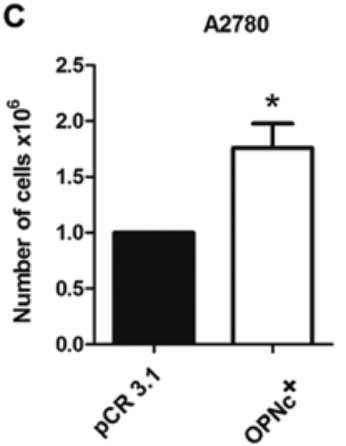

B

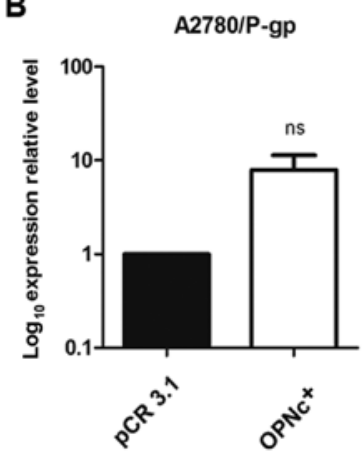

D

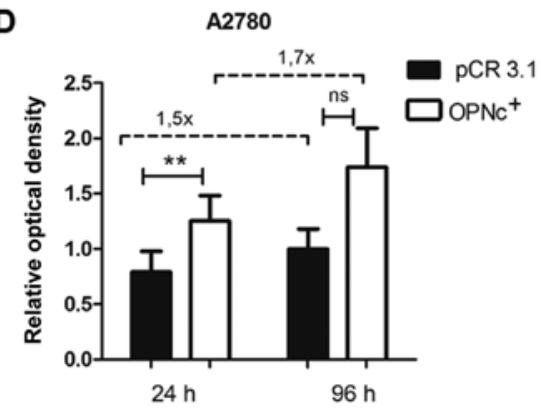

E

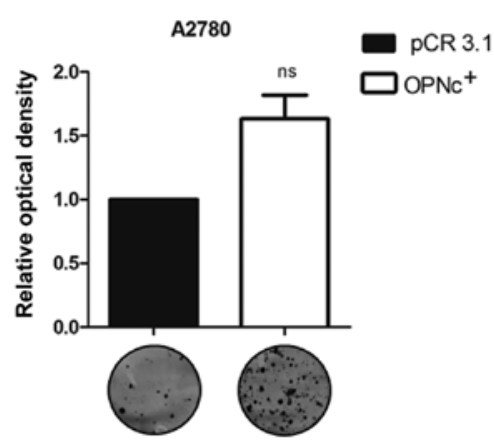

Figure 4. OPNc overexpression promotes cell viability and clonogenicity. A2780 cells were transfected with pCR3.1 or OPNc ${ }^{+}$, and reverse transcription-quan-

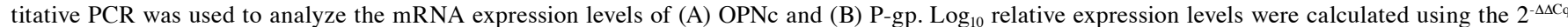
method and normalized to those of $\beta$-actin. OPNc ${ }^{+}$-transfected cells were analyzed for (C) cell viability by trypan blue exclusion analysis and (D) proliferation rates at 24 and $96 \mathrm{~h}$. (E) OPNc+- and pCR3.1-transfected cells were assessed for clonogenicity following 14-day culture by crystal violet staining. Optical density was obtained at $595 \mathrm{~nm}$. ${ }^{*} \mathrm{P} \leq 0.05$ and ${ }^{* *} \mathrm{P} \leq 0.01$; ns, non-significant. OPNc, osteopontin-c isoform; OPNc ${ }^{+}$, OPNc overexpression vector; pCR3.1, empty plasmid; P-gp, P-glycoprotein.

inhibited OPNc expression in OPNc+ cells, increasing cell sensitivity and further decreasing cell viability.

\section{Discussion}

The present study aimed to evaluate whether OPN-SIs were differentially expressed in CDDP-resistant ovarian cancer cells compared with their parental cells. Based on the differential expression patterns, the contribution of OPNc to the modulation of chemoresistance was selected for further investigation in this cell line model. The results demonstrated that the expression levels of the OPNc splice variant were upregulated in ACRP cells compared with those in the parental cell line. Using an OPNc-specific knockdown approach in the ACRP cell line, the present study revealed that OPNc modulated various aspects of drug resistance, such as cell viability, sensitivity to CDDP, and colony formation, as well as the EMT phenotype and the expression of the associated cytokines. A number of these features were also validated by stably overexpressing $\mathrm{OPNc}$ in the A2780 parental cell line.

The results of the present study demonstrated that ACRP cells presented with higher expression levels of OPNc compared with those in A2780 cells. Similarly to these results, previous studies have demonstrated that tOPN expression was induced in human hepatocellular carcinoma cells resistant to CDDP compared with non-resistant cells (11), in colorectal cancer cells resistant to oxaliplatin (13) and in glioma cells resistant to temozolomide (TMZ) and CDDP (15) and alkylphosphocholines (24). In a number of experimental models, tOPN expression levels were upregulated in response to drug treatment $(11,13,15)$. In addition, tOPN knockdown sensitized cells to drug exposure (15). It has also been demonstrated that oncogenic signaling pathways, such as PI3K, NF- $\mathrm{KB} / \mathrm{Bcl}-2$ and $\mathrm{Raf} / \mathrm{MEK} / \mathrm{ERK}$, are activated in response to tOPN overexpression, favoring drug resistance (15). Similarly to tOPN and OPNc, overexpression of other gene products have also 
A

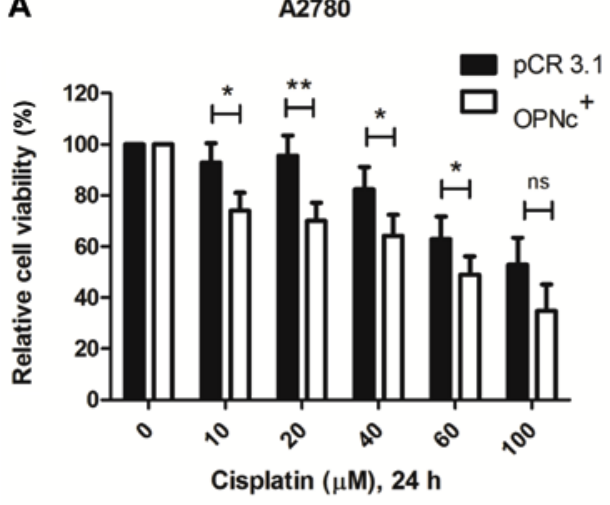

C

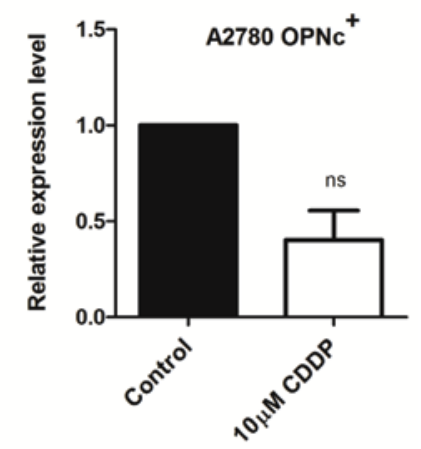

\section{B}

A2780

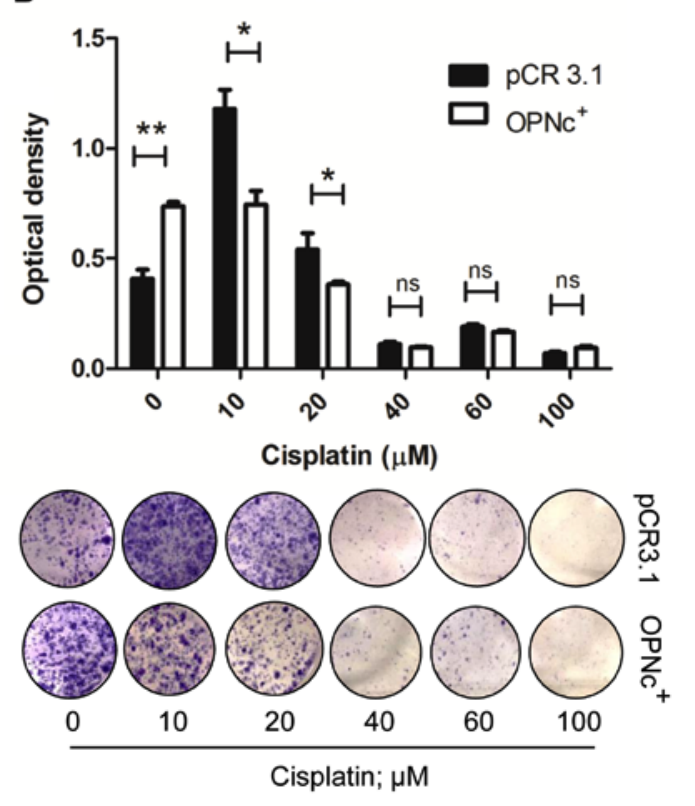

Figure 5. OPNc overexpression sensitizes ovarian cancer cells to cisplatin treatment. (A) Following 24-h transfection, the viability of OPNc ${ }^{+}-$and pCR3.1-transfected cells were analyzed by MTT assay following treatment with increasing concentrations of cisplatin for 24 h. (B) Clonogenicity of OPNc ${ }^{+}-$ and pCR3.1-transfected cells was determined following treatment with increasing concentrations of cisplatin and 14-day culture by crystal violet staining. Optical density was obtained at $595 \mathrm{~nm}$. Images are representative of three independent experiments. (C) A2780 OPNc ${ }^{+}-$and pCR3.1-transfected cells were treated with $10 \mu \mathrm{M}$ cisplatin for $24 \mathrm{~h}$. Cells were then harvested for reverse transcription-quantitative PCR analysis of OPNc expression levels. Relative expression levels were calculated using the $2^{-\Delta \Delta \mathrm{Cq}}$ method and normalized to those of $\beta$-actin. ${ }^{*} \mathrm{P} \leq 0.05$ and ${ }^{* *} \mathrm{P} \leq 0.01$; ns, non-significant. OPNc, osteopontin-c isoform; $\mathrm{OPNc}^{+}, \mathrm{OPNc}$ overexpression vector; pCR3.1, empty plasmid.

been associated with resistance to distinct therapeutic drugs, including GATA binding protein 1 (25), secretory clusterin (26), Her-2 (27) and drug efflux pumps, including P-gp $(28,29)$. Although studies have reported an association between tOPN expression and chemoresistance (10,12-15), the functional roles of specific OPN-SIs in chemoresistant cells have not been previously determined. The results of the present study suggested a potential role for OPNc in chemoresistance in the ACRP and OVCar-8/Dox ${ }^{\mathrm{R}}$ cell line models. In accordance with this hypothesis, our recent study demonstrated that ectopic overexpression of OPNb and OPNc in PC3 prostate cancer cells induced resistance to docetaxel (19), suggesting that overexpression of specific OPN-SIs may differentially contribute to tumor drug resistance. The expression levels of OPNa and OPNb were also upregulated in the ACRP cell line compared with those in A2780 cells in the present study, although at lower levels than OPNc. Future work should evaluate their putative contribution to the resistant phenotype. Notably, in the present study, when OPNc was knocked down, OPNa and $\mathrm{OPNb}$ were upregulated; the indirect effects of OPNc knockdown on the expression of the two other splice variants may be explained by cellular compensation due to low OPNc levels. However, the specific mechanisms by which OPNc may favor chemoresistance are currently unknown. Based on available data regarding the roles of tOPN in several tumor types, we hypothesize that in the experimental model used in the present study, OPNc may activate signaling pathways that contribute to the survival of ovarian tumor cells, favoring increased viability and colony-forming capacity. Similarly, our previous study demonstrated that ectopic OPNc overexpression in the ovarian cancer line OVCar-3 contributed to increased migration, proliferation, cell invasion, independent anchorage and tumor formation in vivo compared with those in OVCar-3 cells transfected with an empty vector (30). It was also observed 


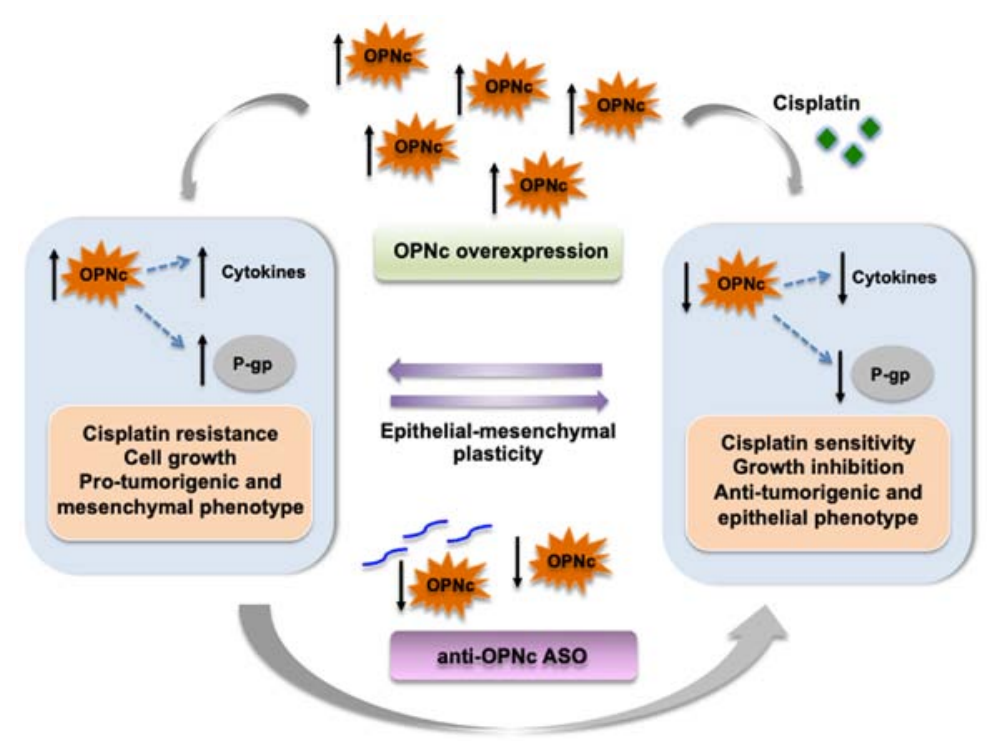

Figure 6. Schematic model for targeting OPNc for cisplatin sensitization in ovarian cancer cells. In ACRP cisplatin-resistant ovarian cancer cells, OPNc upregulation is associated with high P-pg mRNA expression levels, possibly contributing to cisplatin extrusion and drug resistance. Knockdown of OPNc levels using an anti-OPNc ASO resulted in decreased P-gp expression levels and survival rates, as well as inhibited the expression of EMT markers and EMT-related cytokines. By contrast, overexpression of OPNc in the A2780 cell line augmented P-gp expression compared with that in the control cells transfected with the empty expression vector. OPNc-overexpressing cells exhibited increased cell viability rates, which rendered them more sensitive to cisplatin exposure compared with the control cells. These results suggested that OPNc may be a preferential target for the cytotoxic effects of cisplatin in ovarian cancer cells, further reducing cell survival and viability. OPNc, osteopontin-c isoform; ASO, antisense oligonucleotide.

that certain OPNc-dependent protumorigenic functions were mediated by the PI3K/AKT signaling pathway, demonstrating the role of OPNc in ovarian cancer tumor progression through the activation of these signaling pathways (30). The protumorigenic functions of the PI3K/AKT pathway also involve the regulation of survival and apoptosis inhibition, which are associated with drug resistance in ovarian cancer cells $(31,32)$. Therefore, we hypothesize that the inhibition of the PI3K/AKT signaling pathway may be one of the mechanisms underlying the biological effects observed in the OPNc-knockdown cells.

tOPN has been reported to be a key regulator of the EMT (33). The specific mechanism involves the modulation of the expression of transcription factors that contribute to EMT initiation, such as Twist, Snail and Slug (33). Therefore, tOPN favors EMT initiation, which in turn contributes to tumor progression and chemoresistance. Although these findings refer only to tOPN, OPNc may similarly modulate cellular plasticity in the ACRP and OVCar- $8 \operatorname{Dox}^{\mathrm{R}}$ cell lines, further contributing to tumor progression.

tOPN modulated the expression of ILs through binding to their receptors in the tumor microenvironment, contributing to the chemoresistance and tumor progression (10). tOPN has also been demonstrated to bind to the C-C motif chemokine receptor 1 (CCR1), leading to the activation of the $\mathrm{PI} 3 \mathrm{~K} / \mathrm{AKT}$ /hypoxia-inducible factor $1 \alpha$ signaling pathway in hepatocellular carcinoma cell lines (34). The same study also reported that blocking the OPN-CCR1 interaction with a CCR1 antagonist restricted the effects of tOPN on tumor progression and metastatic capacity in this tumor model (34). Therefore, OPNc upregulation may contribute to the increased expression of the cytokines analyzed in the present study through the activation of their corresponding cellular receptors. Indeed, the mRNA expression levels of the GP130 receptor were downregulated in the OPNc-knockdown cells in the present study. Thus, OPNc may act directly and indirectly by promoting the activation of signaling pathways associated with cell survival, inhibition of apoptosis, tumor progression and chemoresistance in ovarian cancer.

In addition to the upregulation of OPNc expression in ACRP cells, the results of the present study demonstrated that P-gp expression levels were upregulated in these cells compared with those in the parental A2780 cells, which has been previously demonstrated in chemoresistant cells in other tumor models (35). Hsieh et al (6) have reported that tOPN modulates the expression of P-gp in a concentration- and time-dependent manner through binding to $\alpha v \beta 3$ integrin (6). However, the contribution of specific OPN-SIs to the regulation of P-gp expression has not been previously addressed. The results of the present study also demonstrated that the upregulation of P-gp in ACRP cells was reversed when OPNc was silenced. Similarly, P-gp expression was induced following OPNc overexpression. These results were in agreement with a previous study, in which knockdown of endogenous tOPN potentiated various P-gp drug substrate-induced apoptosis of prostate cancer cells (6). These results suggest that OPNc may be a modulator of P-gp expression and a potential target to modulate drug efflux by P-gp in cancer cells, facilitating tumor resistance. However, the molecular mechanisms by which tOPN, and specifically OPNc, regulates the expression of $\mathrm{P}-\mathrm{gp}$ remain unknown and should be determined in future studies. High levels of P-gp have been demonstrated to induce TMZ resistance in a glioblastoma (GBM) model (36). Increasing concentrations of $\mathrm{TMZ}$ competed with calcein for P-gp, resulting in reduced efflux in the Adriamycin-resistant DC3F cells; however, various inhibitors of P-gp reversed TMZ resistance in two GBM cell lines by increasing the levels of active caspase-3, suggesting that P-gp may be a key regulator of TMZ resistance in GBM (36). High expression of P-gp has 
been reported to confer resistance to a wide range of structurally and functionally unrelated chemotherapeutic agents (37). In the present study, OPNc was demonstrated to regulate P-gp gene expression, suggesting that targeting the OPNc/P-gp signaling axis may be a potential approach for ovarian cancer cell sensitization to Dox and CDDP.

The results of the present study also demonstrated that OPNc expression levels modulate the survival of drug-resistant ovarian carcinoma cells. In response to OPNc knockdown, the viability of drug-resistant ovarian cancer cells decreased, resulting in higher sensitivity to CDDP and Dox exposure compared with that of the control cells. These results were in accordance to a role of OPNc as a putative novel gene product able to activate the key steps towards resistance to these chemotherapeutic drugs. Similar results have been observed for other proteins that mediated resistance to chemotherapy by stimulating cell survival and inhibiting apoptosis, as well as promoting the cell cycle, including Bcl-2, inhibitor of apoptosis proteins and the heat shock protein family (5). In addition, tOPN has also been described as a modulator of cell survival in response to chemotherapeutic drugs $(10,38)$. In light of these results, among the three OPN-SIs tested in the present study, OPNc may be a key contributor to the cellular processes mediating chemoresistance, such as cell survival and viability, at least in chemoresistant ovarian cancer cells. Future studies should further characterize the targets and pathways by which OPNc may activate cell survival in response to chemotherapeutic drugs.

Following silencing OPNc expression in OVCar-8 Dox ${ }^{\mathrm{R}}$ cells, increased sensitivity to Dox treatment compared with that in the control cells was observed in the present study. Similarly, the results demonstrated decreased viability and colony formation capacity in these cells, along with a modulation in the expression of EMT markers and EMT-related cytokines. Considering similar experimental findings in CDDP- and Dox-resistant cell lines following OPNc silencing, we hypothesize that targeting OPNc may counteract resistance to various chemotherapeutic drugs, similarly to Dox and CDDP. However, further studies should investigate whether these findings can be extended to other drugs.

The results of the present study demonstrated that OPNc regulated the EMT in the ACRP cell line. OPNc knockdown altered the pattern of the intermediate EMT phenotype observed in ACRP cells. These results were in accordance with a putative role of OPNc as a modulator of EMT, which was previously assigned to tOPN in several experimental models, such as in breast cancer, hepatocellular carcinoma, ovarian, gastric and colorectal cancer, as well as melanoma and brain tumors (33). In the lung cancer cell line A549, Huang et al (39) have demonstrated that OPNc overexpression induces increased cell migration and invasion, and alters the expression of EMT markers (decreases E-cadherin and increases vimentin expression) in response to TGF- $\beta$ treatment, indicating that OPNc stimulates cellular plasticity of A549 tumor cells. The roles of OPNc in non-small cell lung cancer cells were mediated by Runt-related transcription factor 2 via a histone deacetylase-dependent pathway, further suggesting that OPNc may be a marker of the EMT and tumor progression in lung cancer. Our previous study in prostate cancer cells resistant to docetaxel supported these results, as cells overexpressing
OPNb or OPNc presented with an EMT-related phenotype typical of chemoresistant cells, as observed in other tumor models (19). The intermediate EMT phenotype observed in CDDP-resistant ACRP ovarian carcinoma cells in the present study was in agreement with a previous study demonstrating that ovarian carcinoma cells present various degrees of EMT (40). An intermediate EMT phenotype has been demonstrated to occur in breast, colon and ovarian carcinoma (41). In addition, the results of the present study demonstrated that OPNc silencing modulate the intermediate EMT phenotype. Notably, ovarian carcinoma has unique biological characteristics, and whether ovarian cancer cells in the primary tumor undergo a complete transition to a mesenchymal state is still under discussion $(40,42)$. In the present study, the EMT does not fully occur in ovarian carcinoma, and is even reversed in tumor cells present in malignant peritoneal and pleural effusions (40). Additionally, Carduner et al (43) have reported that ascites in patients with ovarian cancer undergo a shift toward an unstable intermediate state of the epithelial-mesenchymal spectrum, conferring aggressive cell behavior, depending on the initial epithelial-mesenchymal background (43). Future studies are required to fully characterize the intermediate EMT phenotype in ACRP cells and its association with OPNc expression by further evaluating the protein expression and subcellular localization of EMT markers.

In the present study, OPNc knockdown reverted the expression pattern of EMT-related cytokines in ACRP cells. It has been demonstrated that a number of interleukins or cytokines induce the EMT, including IL- 6 , IL- 8 and TGF- $\beta(44,45)$. The role of cytokines in cancer has been widely studied, particularly in ovarian cancer (46). In this context, certain cytokines have been demonstrated to induce phenotypes consistent with the EMT in transformed epithelial as well as tumor cell lines $(47,48)$. The results of the present study demonstrated that the expression levels of OPNc appeared to modulate the expression of EMT-related cytokines in the drug-resistant cell lines, which in turn induced changes in the expression of EMT markers, as has been previously observed $(44,45)$. A previous study has demonstrated that tOPN modulates cytokine expression, contributing to tumor progression; the interaction between tOPN-IL-6-STAT3 contributes to the invasive and migratory features of osteosarcoma tumor cells, enhancing their metastatic potential (49). Since these results refer to tOPN, the present results are the first to report the role of OPN-SIs, mainly OPNc, in the modulation of the expression of EMT-related cytokines. Further studies are needed to determine the regulatory mechanisms between OPNc and the investigated cytokines in the in vitro chemoresistance models. We hypothesize that upregulation of OPNc may contribute to the increased expression of the cytokines analyzed in the present study through the activation of their corresponding cellular receptors. This was observed to occur for the GP130 receptor, the mRNA levels of which were downregulated in the OPNc-knockdown cells in the present study. Thus, OPNc may act directly and indirectly by promoting the activation of signaling pathways associated with cell survival, inhibition of apoptosis, tumor progression and chemoresistance in ovarian cancer.

To validate the data from OPNc silencing experiments, the present study overexpressed OPNc in the A2780 cell line. 
Cells overexpressing OPNc displayed increased cell viability and colony formation capacity compared with those in cells transfected with the empty expression vector. These data reinforced the pro-survival roles of OPNc in these ovarian cancer cells. Similar to ACRP cells in which OPNc was silenced, A2780 $\mathrm{OPNc}^{+}$cells were also more sensitive to CDDP treatment compared with the control cells. These results suggested that cells overexpressing OPNc may be preferentially targeted for CDDP sensitization. Further corroborating our hypothesis, the results of the present study demonstrated that CDDP downregulated the expression of $\mathrm{OPNc}$ in $\mathrm{OPNc}^{+}$cells. As samples from patients with ovarian cancer exhibit high levels of OPNc (30), and the results of the present study demonstrated that CDDP-resistant cells present upregulated levels of OPNc, we hypothesized that targeting OPNc in ovarian cancer cells with high levels of OPNc may be a promising new therapeutic approach to overcome resistance to CPPD. In addition, combining OPNc silencing with CDDP treatment may possibly enhance cytotoxicity in these resistant cells. One explanation for this effect may be that CDDP, in addition to inhibiting OPNc expression, may also somehow prevent OPNc binding to classical OPN receptors, such as CD44 and integrin heterodimers, which is worth investigating in the future.

The cytotoxicity assays performed in the present study revealed that ACRP cells became more sensitive to CDDP compared with A2780 cells when CDDP concentrations were progressively increased (data not shown). Accordingly, drug adaptation and thus, resistance, are considered to be a dose-dependent condition for certain drug classes and in vitro models. In addition, the present study demonstrated that OPNc silencing in ACRP cells counteracted cell viability and resistance to CDDP. Notably, $\mathrm{OPNc}^{+}$cells exhibited increased sensitivity to CDDP compared with that in the control cells, which may be associated with CDDP-induced OPNc downregulation. In the experimental settings of the present study involving the ACRP cell line which exhibited upregulation of endogenous OPNc, as well as $\mathrm{A} 2780 \mathrm{OPNc}^{+}$cells, targeting OPNc sensitized cells to CDDP. Based on these results, we hypothesized that $\mathrm{OPNc}^{+}$cells may be suitable candidates for novel approaches to CDDP treatment. Translating these findings into the clinical practice, high OPNc expression in patients with ovarian cancer at diagnosis may be a potential predictor for a favorable response to CDDP treatment. This idea follows the rationale reported for other drug targets, such as Her-2 (50-52). In addition, approaches aiming at inducing cell toxicity based on oncogene expression are already used in other tumor models, such as in Her2-positive breast, gastric and esophageal cancer, in which distinct strategies have been proposed to block Her2-induced tumor growth (53-55).

In conclusion, the results of the present study demonstrated that OPNc was upregulated in CDDP-resistant ACRP cells and may serve important roles in modulating various biological processes associated with drug resistance, including cell viability, the EMT phenotype and the expression of EMT-related cytokines. $\mathrm{OPNc}^{+}$cells were more sensitive to CDDP cytotoxicity compared with the negative control cells, suggesting that OPNc overexpression may be a potential strategy for targeted therapy to improve CDDP sensitivity in ovarian cancer cells. Therefore, we propose a model in which OPNc is a key modulator of resistance to CDDP in ovarian cancer cells (Fig. 6). These early insights on the role of OPNc in cancer drug resistance provide a rationale for using this variant as a novel potential molecular target to sensitize ovarian cancer cells to Dox and CDDP treatment.

\section{Acknowledgements}

Not applicable.

\section{Funding}

This study was funded by Fundação de Amparo à Pesquisa do Estado do Rio de Janeiro (grant nos. E-26/210.394/2014, E-26/010.002007/2014 and E-26/203.204/2015), Conselho Nacional de Desenvolvimento Científico e Tecnológico (grant no. 310591/2014-7), Ministério da Sáude, Universidade Federal Fluminense/Pró-Reitoria de Pesquisa e Inovação, the L'Oréal-UNESCO-ABC prize for Women in Science and Fundação do Câncer (Programa de Oncobiologia).

\section{Availability of data and materials}

The datasets used and/or analyzed during the current study are available from the corresponding author on reasonable request.

\section{Authors' contributions}

MCMB participated in the design of the study, performed the majority of the experiments and analyzed the data. LBF participated in the data analysis, reviewed and edited the manuscript. IDSG participated in in the design of the study and data analysis, as well as reviewed and edited the manuscript. LBAR participated in the design of the study and provided support to this work. RCM provided support to this work, reviewed and edited the manuscript. GNDM and ERPG are co-senior authors and equally participated in the design of the study, analyzed and summarized the data, provided financial support, wrote, reviewed and edited of the manuscript. All authors read and approved the final manuscript.

\section{Ethics approval and consent to participate}

Not applicable.

\section{Patient consent for publication}

Not applicable.

\section{Competing interests}

The authors declare that they have no competing interests.

\section{References}

1. International Agency for Research on Cancer (IARC): Global Cancer Observatory. IARC, Lyon, 2020. https://gco.iarc.fr/.

2. Oronsky B, Ray CM, Spira AI, Trepel JB, Carter CA and Cottrill HM: A brief review of the management of platinum-resist ant-platinum-refractory ovarian cancer. Med Oncol 34: 103, 2017.

3. Freimund AE, Beach JA, Christie EL and Bowtell DDL: Mechanisms of drug resistance in high-grade serous ovarian cancer. Hematol Oncol Clin North Am 32: 983-996, 2018. 
4. Gottesman MM, Lavi O, Hall MD and Gillet JP: Toward a better understanding of the complexity of cancer drug resistance. Annu Rev Pharmacol Toxicol 56: 85-102, 2016.

5. Pandey MK, Prasad S, Tyagi AK, Deb L, Huang J, Karelia DN, Amin SG and Aggarwal BB: Targeting cell survival proteins for cancer cell death. Pharmaceuticals (Basel) 9: 11, 2016.

6. Hsieh IS, Huang WH, Liou HC, Chuang WJ, Yang RS and Fu WM: Upregulation of drug transporter expression by osteopontin in prostate cancer cells. Mol Pharmacol 83: 968-977, 2013.

7. Du B and Shim JS: Targeting epithelial-mesenchymal transition (EMT) to overcome drug resistance in cancer. Molecules 21: 965 2016.

8. Elaskalani O, Razak NBA, Falasca M and Metharom P: Epithelial-mesenchymal transition as a therapeutic target for overcoming chemoresistance in pancreatic cancer. World $\mathbf{J}$ Gastrointest Oncol 9: 37-41, 2017.

9. He Y, Xie H, Yu P, Jiang S and Wei L: FOXC2 promotes epithelial-mesenchymal transition and cisplatin resistance of non-small cell lung cancer cells. Cancer Chemother Pharmacol 82: 1049-1059, 2018.

10. Gimba ERP, Brum MCM and Nestal De Moraes G: Full-length osteopontin and its splice variants as modulators of chemoresistance and radioresistance (Review). Int J Oncol 54: 420-430, 2019.

11. Ding K, Fan L, Chen S, Wang Y, Yu H, Sun Y, Yu J, Wang L, Liu X and Liu Y: Overexpression of osteopontin promotes resistance to cisplatin treatment in HCC. Oncol Rep 34: 3297-3303, 2015.

12. Grbčić P, Tomljanović I, Klobučar M, Kraljević Pavelić S, Lučin K and Sedić M: Dual sphingosine kinase inhibitor SKI-II enhances sensitivity to 5-fluorouracil in hepatocellular carcinoma cells via suppression of osteopontin and FAK/IGF-1R signalling. Biochem Biophys Res Commun 487: 782-788, 2017.

13. Ng L, Wan T, Chow A, Iyer D, Man J, Chen G, Yau TC, Lo O, Foo CC, Poon JT, et al: Osteopontin overexpression induced tumor progression and chemoresistance to oxaliplatin through induction of stem-like properties in human colorectal cancer. Stem Cells Int 2015: 247892, 2015.

14. Pang H, Cai L, Yang Y, Chen X, Sui G and Zhao C: Knockdown of osteopontin chemosensitizes MDA-MB-231 cells to cyclophosphamide by enhancing apoptosis through activating p38 MAPK pathway. Cancer Biother Radiopharm 26: 165-173, 2011.

15. Qian C, Li P, Yan W, Shi L, Zhang J, Wang Y, Liu H and You Y: Downregulation of osteopontin enhances the sensitivity of glioma U251 cells to temozolomide and cisplatin by targeting the NF- $\kappa \mathrm{B} / \mathrm{Bcl}-2$ pathway. Mol Med Rep 11: 1951-1955, 2015.

16. Gimba ER and Tilli TM: Human osteopontin splicing isoforms: Known roles, potential clinical applications and activated signaling pathways. Cancer Lett 331: 11-17, 2013.

17. Hui T, Sørensen ES and Rittling SR: Osteopontin binding to the alpha 4 integrin requires highest affinity integrin conformation, but is independent of post-translational modifications of osteopontin. Matrix Biol 41: 19-25, 2015

18. Kazanecki CC, Uzwiak DJ and Denhardt DT: Control of osteopontin signaling and function by post-translational phosphorylation and protein folding. J Cell Biochem 102: 912-924, 2007.

19. Nakamura KDM, Tilli TM, Wanderley JL, Palumbo A Jr, Mattos RM, Ferreira AC, Klumb CE, Nasciutti LE and Gimba ER: Osteopontin splice variants expression is involved on docetaxel resistance in PC3 prostate cancer cells. Tumour Biol 37: 2655-2663, 2016

20. Bastos ACSF, Blunck CB, Emerenciano $M$ and Gimba ERP Osteopontin and their roles in hematological malignancies: Splice variants on the new avenues. Cancer Lett 408: 138-143, 2017.

21. Santoro JC, Bastos ACSF, Gimba ERP and Emerenciano M Reinforcing osteopontin as a marker of central nervous system relapse in paediatric B-cell acute lymphoblastic leukaemia: SPP1 splice variant 3 in the spotlight. Br J Haematol 186: e88-e91, 2019.

22. Sherman-Baust CA, Weeraratna AT, Rangel LBA, Pizer ES, Cho KR, Schwartz DR, Shock T and Morin PJ: Remodeling of the extracellular matrix through overexpression of collagen VI contributes to cisplatin resistance in ovarian cancer cells. Cancer Cell 3: 377-386, 2003

23. Livak KJ and Schmittgen TD: Analysis of relative gene expression data using real-time quantitative PCR and the 2(-Delta Delta C(T)) Method. Methods 25: 402-408, 2001

24. Yosifov DY, Reufsteck C, Konstantinov SM and Berger MR: Interleukin-6, osteopontin and Raf/MEK/ERK signaling modulate the sensitivity of human myeloma cells to alkylphosphocholines. Leuk Res 36: 764-772, 2012.
25. Caldwell JT, Edwards H, Dombkowski AA, Buck SA, Matherly LH, Ge Y and Taub JW: Overexpression of GATA1 confers resistance to chemotherapy in acute megakaryocytic Leukemia. PLoS One 8: e68601, 2013.

26. Niu ZH, Wang Y, Chun B, Li CX and Wu L: Secretory clusterin (sCLU) overexpression is associated with resistance to preoperative neoadjuvant chemotherapy in primary breast cancer. Eur Rev Med Pharmacol Sci 17: 1337-1344, 2013

27. Wilks ST: Potential of overcoming resistance to HER2-targeted therapies through the PI3K/Akt/mTOR pathway. Breast 24: $548-555,2015$

28. Montazami N, Aghapour M, Farajnia S and Baradaran B: New insights into the mechanisms of multidrug resistance in cancers. Cell Mol Biol (Noisy-le-grand) 61: 70-80, 2015.

29. Yang X, Yi C, Luo N and Gong C: Nanomedicine to overcome cancer multidrug resistance. Curr Drug Metab 15: 632-649, 2014.

30. Tilli TM, Franco VF, Robbs BK, Wanderley JL, da Silva FR, de Mello KD, Viola JP, Weber GF and Gimba ER: Osteopontin-c splicing isoform contributes to ovarian cancer progression. Mol Cancer Res 9: 280-293, 2011.

31. Lee S, Choi EJ, Jin C and Kim DH: Activation of PI3K/Akt pathway by PTEN reduction and PIK3CA mRNA amplification contributes to cisplatin resistance in an ovarian cancer cell line. Gynecol Oncol 97: 26-34, 2005.

32. Zhou B, Sun C, Li N, Shan W, Lu H, Guo L, Guo E, Xia M, Weng D, Meng L, et al: Cisplatin-induced CCL5 secretion from CAFs promotes cisplatin-resistance in ovarian cancer via regulation of the STAT3 and PI3K/Akt signaling pathways. Int J Oncol 48: 2087-2097, 2016.

33. Kothari AN, Arffa ML, Chang V, Blackwell RH, Syn WK, Zhang J, Mi Z and Kuo PC: Osteopontin-A Master Regulator of Epithelial-Mesenchymal Transition. J Clin Med 5: 5, 2016.

34. Zhu Y, Gao XM, Yang J, Xu D, Zhang Y, Lu M, Zhang Z, Sheng YY, Li JH, Yu XX, et al: C-C chemokine receptor type 1 mediates osteopontin-promoted metastasis in hepatocellular carcinoma. Cancer Sci 109: 710-723, 2018.

35. Breier A, Gibalova L, Seres M, Barancik M and Sulova Z: New insight into p-glycoprotein as a drug target. Anticancer Agents Med Chem 13: 159-170, 2013.

36. Munoz JL, Walker ND, Scotto $\mathrm{KW}$ and Rameshwar $\mathrm{P}$ Temozolomide competes for P-glycoprotein and contributes to chemoresistance in glioblastoma cells. Cancer Lett 367: 69-75, 2015.

37. Robey RW, Pluchino KM, Hall MD, Fojo AT, Bates SE and Gottesman MM: Revisiting the role of ABC transporters in multidrug-resistant cancer. Nat Rev Cancer 18: 452-464, 2018

38. Luo SD, Chen YJ, Liu CT, Rau KM, Chen YC, Tsai HT, Chen CH and Chiu TJ: Osteopontin involves cisplatin resistance and poor prognosis in oral squamous cell carcinoma. BioMed Res Int 2015: 508587, 2015.

39. Huang J, Chang S, Lu Y, Wang J, Si Y, Zhang L, Cheng S and Jiang WG: Enhanced osteopontin splicing regulated by RUNX2 is HDAC-dependent and induces invasive phenotypes in NSCLC cells. Cancer Cell Int 19: 306, 2019.

40. Davidson B, Tropé CG and Reich R: Epithelial-mesenchymal transition in ovarian carcinoma. Front Oncol 2: 33, 2012.

41. Huang RYJ, Wong MK, Tan TZ, Kuay KT, Ng AH, Chung VY, Chu YS, Matsumura N, Lai HC, Lee YF, et al: An EMT spectrum defines an anoikis-resistant and spheroidogenic intermediate mesenchymal state that is sensitive to e-cadherin restoration by a src-kinase inhibitor, saracatinib (AZD0530). Cell Death Dis 4 : e915, 2013.

42. Chebouti I, Kasimir-Bauer S, Buderath P, Wimberger P, Hauch S, Kimmig R and Kuhlmann JD: EMT-like circulating tumor cells in ovarian cancer patients are enriched by platinum-based chemotherapy. Oncotarget 8: 48820-48831, 2017.

43. Carduner L, Leroy-Dudal J, Picot CR, Gallet O, Carreiras F and Kellouche S: Ascites-induced shift along epithelial-mesenchymal spectrum in ovarian cancer cells: Enhancement of their invasive behavior partly dependant on $\alpha v$ integrins. Clin Exp Metastasis 31: 675-688, 2014.

44. Techasen A, Loilome W, Namwat N, Dokduang H, Jongthawin J and Yongvanit P: Cytokines released from activated human macrophages induce epithelial mesenchymal transition markers of cholangiocarcinoma cells. Asian Pac J Cancer Prev 13 (Suppl): 115-118, 2012.

45. Sistigu A, Di Modugno F, Manic G and Nisticò P: Deciphering the loop of epithelial-mesenchymal transition, inflammatory cytokines and cancer immunoediting. Cytokine Growth Factor Rev 36: 67-77, 2017. 
46. Rezaeifard S, Razmkhah M, Robati M, Momtahan M and Ghaderi A: Cytokines, chemokines, and chemokine receptors quantitative expressions in patients with ovarian cancer. Iran J Med Sci 40: 225-232, 2015.

47. Sullivan NJ, Sasser AK, Axel AE, Vesuna F, Raman V, Ramirez N, Oberyszyn TM and Hall BM: Interleukin-6 induces an epithelial-mesenchymal transition phenotype in human breast cancer cells. Oncogene 28: 2940-2947, 2009.

48. Fernando RI, Hamilton DH, Huang B and Palena C: Interleukin-8 drives epithelial-mesenchymal transition of human carcinomas. J Immunother Cancer 1 (S1): 187, 2013.

49. Zhang C, Ma K and Li WY: IL-6 promotes cancer stemness and oncogenicity in U2OS and MG-63 osteosarcoma cells by upregulating the OPN-STAT3 pathway. J Cancer 10: 6511-6525, 2019.

50. Learn PA, Yeh IT, McNutt M, Chisholm GB, Pollock BH, Rousseau DL Jr, Sharkey FE, Cruz AB and Kahlenberg MS: HER-2/neu expression as a predictor of response to neoadjuvant docetaxel in patients with operable breast carcinoma. Cancer 103 2252-2260, 2005.

51. Omarini C, Bettelli S, Caprera C, Manfredini S, Caggia F, Guaitoli G, Moscetti L, Toss A, Cortesi L, Kaleci S, et al: Clinical and molecular predictors of long-term response in HER2 positive metastatic breast cancer patients. Cancer Biol Ther 19: 879-886, 2018.
52. Kwon MJ, Soh JS, Lim SW, Kang HS and Lim H: HER2 as a limited predictor of the therapeutic response to neoadjuvant therapy in locally advanced rectal cancer. Pathol Res Pract 215: 910-917, 2019.

53. Gerson JN, Skariah S, Denlinger CS and Astsaturov I: Perspectives of HER2-targeting in gastric and esophageal cancer. Expert Opin Investig Drugs 26: 531-540, 2017.

54. El Dika I and Ilson DH: Current and future therapies for targeting HER 2 mutations in gastrointestinal cancer. Expert Rev Anticancer Ther 18: 1085-1092, 2018.

55. Dong Y,Li W, Gu Z, Xing R, Ma Y,Zhang Q and Liu Z: Inhibition of HER2-positive breast cancer growth by blocking the HER2 signaling pathway with HER2-glycan-imprinted nanoparticles. Angew Chem Int Ed Engl 58: 10621-10625, 2019.

This work is licensed under a Creative Commons Attribution-NonCommercial-NoDerivatives 4.0 International (CC BY-NC-ND 4.0) License. 